TITLE:

\title{
Age-related muscle atrophy in the lower extremities and daily physical activity in elderly women.
}

\author{
$\operatorname{AUTHOR}(S):$ \\ Ikezoe, Tome; Mori, Natsuko; Nakamura, \\ Masatoshi; Ichihashi, Noriaki
}

\section{CITATION:}

Ikezoe, Tome ... [et al]. Age-related muscle atrophy in the lower extremities and daily physical activity in elderly women.. Archives of gerontology and geriatrics 2011, 53(2): e153-e157

ISSUE DATE:

2011-09

URL:

http://hdl.handle.net/2433/143669

\section{RIGHT:}

(C) 2011 Elsevier Ireland.; この論文は出版社版でありません。引用の際 には出版社版をご確認ご利用ください。; This is not the published version. Please cite only the published version. 
$M-2111(R)$

Age-related muscle atrophy in the lower extremities and daily physical activity

in elderly women

Tome Ikezoe*", Natsuko Mori, Masatoshi Nakamura, Noriaki Ichihashi

Human Health Sciences, Graduate School of Medicine, Kyoto University,

53 Shogoin-Kawahara-cho, Sakyo-ku, Kyoto 606-8507, Japan 
*Corresponding author:

Phone: +(81-75)-751-3964

Fax: $+(81-75)-751-3909$

E-mail: ikezoe@hs.med.kyoto-u.ac.jp

Article history:

Received: 26 April 2010.

Received in revised form: 31 July 2010.

Accepted: 3 August 2010. 


\section{Abstract}

This study investigated the relationship between age-related declines in muscle

thickness of the lower extremities and daily physical activity in elderly women. The subjects comprised 20 young women and 17 elderly women residing in a nursing home. Lower-limb muscle thickness was measured by B-mode ultrasound with the following ten muscles; gluteus maximus, gluteus medius, gluteus minimus, psoas major, rectus femoris, vastus lateralis, vastus intermedius, biceps femoris, gastrocnemius and soleus. Daily physical activity was evaluated using life-space assessment (LSA) which assessed the life-space level, degree of independence, and frequency of attainment. Muscle thickness in the gluteus maximus, gluteus medius, gluteus minimus, psoas major, rectus femoris, vastus lateralis, vastus intermedius, biceps femoris and gastrocnemius, but not soleus, was significantly greater in the young group than the elderly group. The greatest rates of age-related loss of skeletal muscle mass in the lower limbs showed in the psoas major, while the smallest loss 
showed in soleus muscle. Only the gluteus medius was significantly associated with the LSA score $(r=0.528, p<0.05)$ in elderly women. These results suggest that the reduction in skeletal mass with age is smaller in soleus muscle, and that the age-related decline in gluteus medius muscle is influenced by daily physical activity.

Keywords: Age-related muscle atrophy, Ultrasonographic assessment, Lower limb muscles, daily physical activity, elderly women

\section{Introduction}

Skeletal muscle mass decreases with aging, and by 30-50\% between the ages of 40 and 80 years (Allen et al.,1960; Young et al.,1985; Lexell et al.,1988; Akima et al.,2001). The muscle atrophy is reported to be greater in the lower limbs than upper limbs (Bemben et al., 1991; Brooks and Faulkner, 1994; Janssen et al., 2000). 
Techniques for estimating skeletal muscle mass in vivo include magnetic resonance imaging (MRI), computed tomography $(\mathrm{CT})$, anthropometry, bioelectrical impedance analysis (BIA) and ultrasound. Ultrasound is particularly useful because it is safe, noninvasive, and portable. Strong correlations have been reported between muscle thickness measured by B-mode ultrasound and site-matched skeletal muscle mass measured by MRI (Walton et al., 1997; Dupont et al., 2001; Fukunaga et al., 2001; Miyatani et al., 2004; Sanada et al., 2006). Therefore, it is plausible to use muscle thickness measurements to estimate muscle size and degree of muscle atrophy.

In recent years, there have been many studies on the age-related atrophy of lower limb muscles by ultrasonographic measurement of the muscle thickness. However, in these studies, the quadriceps (Reimers et al., 1998; Kubo et al., 2003a, 2003b; Arts et al., 2010) and triceps surae muscles (Reimers et al., 1998; Kubo et al., 2003a, 2003b; Fujiwara et al., 2010) have been mainly evaluated. There have been 
only a few studies on age-related changes in individual muscles constituting lower limb muscles including those around the hip joint.

Loss of skeletal muscle mass is part of the aging process and exacerbated by inactivity (Sandler et al., 1991). The age-associated reduction in physical activity may be at least partially responsible for the change in muscle distribution with age, given that it would lead to greater muscle loss in the lower limbs, that is, those muscles are required for most common daily activities like walking, climbing stairs etc. Yazawa et al. (2007) reported a significant correlation between daily physical activity measured with an accelerometer and lower body muscle mass measured by bioelectrical impedance analysis (BIA) in the middle-aged and elderly. Park et al. (2010) demonstrated that muscle mass in the lower extremities measured by whole-body dual X-ray absorptiometer (DXA) was associated with physical activity such as daily step count. 
The aim of this study was twofold; first, to investigate the influences of age on skeletal muscle mass in the lower extremities using ultrasound and, second, to examine the influence of daily physical activity on muscle atrophy in elderly women.

\section{Subjects and methods}

\subsection{Subjects}

The subjects comprised 20 healthy young women with a mean age of $19.8 \pm 0.8$ years $( \pm$ S.D.) and 17 elderly women with a mean age of $83.2 \pm 6.2$ years. All the elderly subjects were residents of nursing home in Kyoto, Japan, and so lived under the same conditions. Table 1 shows the characteristics of the subjects. The elderly subjects were able to ambulate independently or with an assistive device, did not have an unstable physical condition, and did not demonstrate severe dementia. Physical dysfunctions such as acute neurological impairment (acute stroke, parkinson's disease, paresis of 
the lower limbs) or severe musculoskeletal impairment, that might affect mobility in daily activities, were also excluded.

The subjects were informed about the study procedures before testing and provided a written informed consent before participating. The study was approved by Kyoto University Graduate School and Faculty of Medicine Ethics Committee.

\subsection{Muscle thickness measurements}

Lower-limb muscle thickness on the longitudinal plane, was measured using B-mode ultrasound imaging (LOGIQ Book Xp; GE Healthcare Japan, Tokyo, Japan), with an 8-MHz transducer.

A previous study has shown the reliability of the ultrasound technique for measuring muscle thickness (Abe et al., 1994; Reeves et al., 2004; Kellis et al., 2009; Thoirs and English, 2009). Ten muscles were examined in the lower extremities on the

right side; gluteus maximus, gluteus medius, gluteus minimus, psoas major, rectus 
femoris, vastus lateralis, vastus intermedius, biceps femoris, gastrocnemius and soleus. Measurement positions and measurement sites for each muscle are shown in Table 2. During the examination, care was taken to maintain the same standardized position of the subjects and the exact location of the transducer. In order to improve acoustic coupling, a water-soluble transmission gel was placed over the scan head.

\subsection{Daily physical activity}

Daily physical activity was evaluated using LSA, described by Baker et al. (2003)

to have good predictive validity for physical performance and self-reported function and high test-retest reliability. LSA assesses the life-space level, degree of independence, and frequency of attainment over the 4 weeks preceding an assessment. It also measures mobility based on the distance a person reports covering during the 4 weeks preceding the assessment. 
Questions establish movement to specific life-space levels ranging from within one's dwelling to beyond one's town with the following five-range classification: (1) to other rooms of your home besides the room where you sleep; (2) to an area outside your home such as your porch or garage; (3) to places in your neighborhood, other than your own yard; (4) to places outside your neighborhood but within your town; (5) to places outside your town.

For each level, persons were asked how many days a week they attained that level and whether they needed help from another person or from assistive devices. Values were computed for each level by multiplying the life-space level (1-5), the degree of independence $(2=$ if independent; i.e., no assistance from persons or equipment was reported, $1.5=$ if equipment was used, and $1=$ if personal assistance was reported), and the frequency of attainment $(1=$ less than once a week, $2=1-3$ times a week, $3=4-6$ times a week, and $4=$ daily). The level-specific values were summed (possible total score $=120$ ). 


\subsection{Statistical analyses}

All data are presented as the mean \pm S.D. Differences in muscle thickness

between the young and elderly groups were examined using Student's t- test (two-sample t-test).

We calculated the muscle thickness in the elderly as a percentage of the mean in the young using the following formula, and analyzed this rate for each muscle employing the multiple comparison method.

the $\%$ of muscle thickness =

(muscle thickness in the elderly/the mean in the young) $\times 100$

Furthermore, Spearman's correlation coefficient was used to investigate the relationship between age-related declines in muscle thickness of the lower extremities and daily physical activity in elderly women. All data were analyzed using SPSS 
(version 17.0; SPSS Japan Inc., Tokyo, Japan). Significance was recognized at $p<$

0.05 .

\section{Results}

Table 3 shows the mean and standard deviation values of muscle thickness for

the young and elderly groups. When the muscle thickness was compared between the

young and elderly, all muscles except the soleus muscle, that is, gluteus maximus,

gluteus medius, gluteus minimus, psoas major, rectus femoris, vastus lateralis, vastus

intermedius, biceps femoris and gastrocnemius were significantly thinner in the elderly.

There was no significant difference between the groups in the thickness of the soleus

muscle (young group $=34.5 \pm 6.1 \mathrm{~mm}$ vs. elderly group $=29.7 \pm 7.9 \mathrm{~mm}$ ).

The rate of muscle thickness in the elderly was the highest for the soleus muscle( $86.1 \%)$, followed in order by the vastus intermedius $(74.8 \%)$, rectus femoris (72.9\%), gastrocnemius (68.1\%), gluteus medius (63.7\%), vastus lateralis $(63.0 \%)$, 
gluteus minimus (62.8\%), gluteus maximus (62.0\%), biceps femoris $(48.9 \%)$ and psoas major (47.2\%). Multiple comparisons used to compare percentages of muscle thickness in the elderly showed the soleus, vastus intermedius and rectus femoris to have significantly greater values than the psoas major and biceps femoris (Table 4).

The thickness of the gluteus medius was significantly associated with the LSA score $(r=0.528, p<0.05)$ in elderly women, whereas no correlation was found between LSA score and the thickness of the gluteus maximus, gluteus minimus, psoas major, rectus femoris, vastus lateralis, vastus intermedius, biceps femoris, gastrocnemius and soleus (Table 5).

\section{Discussion}

The main findings of this study were that age-related muscle atrophy was smallest for soleus muscle among 10 muscles of the lower limbs, and that age-related declines in gluteus medius muscle was associated with daily physical activity. To our 
knowledge, this is the first report showing age-related declines in individual muscles

constituting lower limb muscles including those around hip, knee and ankle joint and the relationship between age-related changes in muscle thickness and daily physical activity in elderly women.

Generally, muscle mass in the lower extremities shows substantial declines with age. In this study, the measurement of the thickness of 10 muscles of the lower limbs showed the smallest degree of atrophy of the soleus muscle without any significant difference in the soleus muscle thickness between the young and elderly. As for age-related changes in the muscle mass of triceps surae muscle, Fujiwara et al. (2010) found that the gastrocnemius was significantly thinner in elderly women than young women, while the thickness of the soleus muscle did not differ among these groups. Morse et al. (2005) also reported that physiological cross-sectional area (PCSA) values measured by MRI for the lateral and medial heads of gastrocnemius muscles were $19 \%$ and $14.5 \%$ smaller in elderly males $(n=12)$ than young males $(n=15)$, 
whereas a non-significant reduction of $4.4 \%$ was observed in the soleus PCSA. In this way, some studies have shown slight age-related changes in the soleus muscle, focusing on the triceps surae muscle alone. It is of interest that this study, in which all lower muscles including those around the hip, knee, and ankle were evaluated, also showed only slight atrophy of the soleus muscle.

The relatively small atrophy of soleus muscle, may be because of the different postural role and fiber-type characteristics of lower body muscles. It has been reported that the mean percentage of type I fibers is $86.4 \%$ in soleus, $43.5 \%$ in gastrocnemius, $42.8 \%$ in rectus femoris, $37.8 \%$ in vastus lateralis and $52.4 \%$ in gluteus maximus (Johnson et al., 1973). In general, greater rates of age-related loss occur among type II fibers, while only moderate losses occur among type I fibers (Lexell et al., 1988; Doherty et al., 1993; Roos et al., 1997). The postural role of the soleus, may protect this muscle from marked reductions with aging given its predominant type I composition, whereas the gastrocnemius muscles which are primarily locomotor 
muscles with a higher type II fiber content may be more susceptible to atrophy as a result of the neuropathic processes associated with aging.

The rate of muscle thickness in the elderly was the lowest for psoas major muscle, suggesting particularly marked age-related changes in this muscle among lower limb muscles. Takahashi et al. (2006) reported that the decline in cross-sectional area (CSA) measured by $\mathrm{MRI}$ due to aging was greater in psoas major than quadriceps femoris, and that the average CSA of psoas major and quadriceps femoris muscles for the 70 s age group compared with the 20s age group was 0.798 and 0.892 , respectively. The results of the present study were consistent with those of Takahashi et al. (2006), because our study indicated that the decline in muscle thickness was greater in psoas major than soleus and quadriceps femoris muscles such as rectus femoris and vastus intermedius.

The rate was the second lowest for the biceps femoris muscle. Previous study has shown that middle-aged male athletes who habitually run at a high level have more 
muscle thickness of the hamstrings than do middle-aged men who run at an intermediary level or do not run at all (Oguri et al., 2004). Furthermore, changes in EMG amplitude in the iliacus and psoas muscles among hip flexor muscles showed a marked increase from walking to running, perhaps because greater activation of the psoas major is required during running (Andersson et al., 1997). This may be due to marked decreases in the sizes of hamstring and psoas major muscles important for running because of decreased opportunities to run in the elderly.

Our analyses of the correlation between muscle thickness and daily physical activity in the elderly group indicated that only the gluteus medius was significantly associated with the LSA score. The gluteus medius is partially responsible for medial-lateral stability of the hip, and if inhibited, would affect the control of the medial-lateral displacement component of functional performance. Concerning the stepping response to perturbation, Maki and Mcllroy (2006) described that the elderly cannot maintain their balance by taking one step when they lose balance and are 
about to fall, and tend to fall laterally, suggesting decreased functions of the muscles around the hip joint such as the gluteus medius muscle as one of the main causes of this instability. Tirosh et al. (2005) reported that the muscle activity of the gluteus medius muscle, which is necessary for control when people stop during walking, was lower in the elderly than in the young, suggesting that the difficulty in stopping suddenly to avoid danger in the elderly is partly due to decreased activity of this muscle.

Our results suggested that decreased stability and fear of falling with a loss of confidence in functional performance, may lead to decreased physical activity and restrictions on physical behavior among elderly persons with muscle loss of the gluteus medius, even among those able to ambulate independently. Furthermore, functional restrictions on daily activities can lead to even more physical inactivity and further declines in muscle mass.

The soleus muscle thickness, showing no difference between the young and elderly, was not associated with the level of activity in daily life. These results suggest 
that the size of the soleus muscle is maintained irrespective of the range of locomotion or frequency of activity in daily life in the elderly who can walk unassisted.

No correlation was observed between daily physical activity and muscle mass of the psoas major which showed the largest magnitude of decline in muscle thickness of the lower extremity. The size of the psoas major muscle is considered to be closely related to the locomotory capacity such as walking and stair climbing in the elderly (Kim et al., 2000; Masuda et al., 2002). As a result of this study, the psoas major muscle showed marked age-related atrophy irrespective of the level of walking in daily life. Kim et al. (2001) found that the CSA of the psoas major was significantly higher in elderly athletes who long continued to train than in the untrained elderly. It seems that higher intensity activities than common daily activities like walking or climbing stairs may be required in order to prevent marked age-related muscular atrophy of psoas major. 
The limitations of this study were as follows. One limitation was measurement of the muscle thickness as the amount of lower limb muscles. In general, the muscle cross-sectional area is considered to reflect the muscle strength. In this study, the muscle thickness, i.e., the longitudinal axis, was measured, but the obtained measurement values did not reflect the transverse axis. Other limitations were the small number of subjects, and the inclusion of only elderly females who are capable of unassisted locomotion. In the future, muscular atrophy should be analyzed in subjects including the elderly with locomotor disability, and the association between the degree of age-related muscular atrophy in the lower limbs and the locomotor capacity should be clarified.

\section{Conclusions}

We evaluated age-related muscular atrophy employing an ultrasonographic method, and observed relatively slight atrophy of the soleus muscle and marked 
atrophy of the psoas major muscle among lower limb muscles in elderly females who can walk without assistance. There was no association between the degree of atrophy of the soleus or psoas major muscle and activities in daily life. However, only the thickness of the gluteus medium muscle was associated with daily life activities.

Conflict of interest statement: None.

\section{References}

Abe, T., Kondo, M., Kawakami, Y., Fukunaga, T., 1994. Prediction equations for body composition of Japanese adults by B-mode ultrasound. Am. J. Hum. Biol. 6, 161-170.

Akima, H., Kano, Y., Enomoto, Y., Ishizu, M., Okada, M., Oishi, Y., Katsuta, S., Kuno, S., 2001. Muscle function in 164 men and women aged 20-84 yr. Med. Sci.

Sports Exerc. 33, 220-226. 
Allen, T.H., Andersen, E.C., Langham, W.H., 1960. Total body potassium and gross body composition in relationship to age. J. Gerontol. 15, 348-357.

Andersson, E.A., Nilsson, J., Thorstensson, A., 1997. Intramuscular EMG from the hip flexor muscles during human locomotion. Acta. Physiol. Scand. 161, 361-370.

Arts, I.M., Pillen, S., Schelhaas, H.J., Overeem, S., Zwarts, M.J., 2010. Normal values for quantitative muscle ultrasonography in adults. Muscle Nerve 41, 32-41.

Baker, P.S., Bodner, E.V., Allman, R.M., 2003. Measuring life-space mobility in community-dwelling older adults. J. Am. Geriatr. Soc. 51, 1610-1614.

Bemben, M.G., Massey, B.H., Bemben, D.A., Misner, J.E., Boileau, R.A., 1991. Isometric force production as a function of age in healthy 20 - to 74 -yr-old men. Med. Sci. Sports Exerc. 23, 1302-1310.

Brooks, S.V., Faulkner, J.A., 1994. Skeletal Muscle Weakness in Old Age: Underlying Mechanisms. Med. Sci. Sports Exerc. 26, 432-439. 
Doherty, T.J., Vandervoort, A.A., Brown, W.F., 1993. Effects of Ageing on the Motor

Unit: A Brief Review. Can. J. Appl. Physiol. 18, 331-358.

Dupont, A.C., Sauerbrei, E.E., Fenton, P.V., Shragge, P.C., Loeb, G.E., Richmond, F.J., 2001. Real-time sonography to estimate muscle thickness: comparison with MRI and CT. J. Clin. Ultrasound 29, 230-236.

Fujiwara, K., Asai, H., Toyama, H., Kunita, K., Yaguchi, C., Kiyota, N., Tomita, H., Jacobs, J.V., 2010. Changes in muscle thickness of gastrocnemius and soleus associated with age and sex. Aging Clin. Exp. Res. 22, 24-30.

Fukunaga, T., Miyatani, M., Tachi, M., Kouzaki, M., Kawakami, Y., Kanehisa, H., 2001. Muscle volume is a major determinant of joint torque in humans. Acta Physiol.

Scand. 172, 249-255.

Janssen, I., Heymsfield, S.B., Wang, Z., Ross, R., 2000. Skeletal muscle mass and distribution in 468 men and women aged 18-88 yr. J. Appl. Physiol. 89, 81-88. 
Johnson, M.A., Polgar, J., Weightman, D., Appleton, D., 1973. Data on the distribution of fibre types in thirty-six human muscles. An autopsy study. J. Neurol. Sci. 18, 111-129.

Kellis, E., Galanis, N., Natsis, K., Kapetanos, G., 2009. Validity of architectural properties of the hamstring muscles: correlation of ultrasound findings with cadaveric dissection. J. Biomech. 42, 2549-2554.

Kim, D.J., Kuno, S., Soma, R., Masuda, K., Ishizu, M., Adachi, T., Nishijima, T., Okada, M., 2000. Relationship between reduction of hip joint and thigh muscle and walking ability in elderly people. Jpn. J. Phys. Fit. Sport 49, 589-596.

Kim, D.J., Ohshima, T., Baba, S., Yasuda, T., Adachi, K., Katuta, S., Okada, M., Kuno, S., 2001. Effect of long-term exercise on walking ability in elderly people. Jpn. J. Phys. Fit. Sport 50, 149-157. 
Kubo, K., Kanehisa, H., Azuma, K., Ishizu, M., Kuno, S.Y., Okada, M., Fukunaga, T., 2003a. Muscle architectural characteristics in young and elderly men and women. Int. J. Sports Med. 24, 125-130.

Kubo, K., Kanehisa, H., Azuma, K., Ishizu, M., Kuno, S.Y., Okada, M., Fukunaga, T., 2003b. Muscle architectural characteristics in women aged 20-79 years. Med. Sci. Sports Exerc. 35, 39-44.

Lexell, J., Taylor, C.C., Sjostrom, M., 1988. What is the cause of the ageing atrophy? Total number, size and proportion of different fiber types studied in whole vastus lateralis muscle from 15- to 83-year-old men. J. Neurol. Sci. 84, 275-294.

Maki, B.E., Mcllroy, W.E., 2006. Control of rapid limb movements for balance recovery: age-related changes and implications for fall prevention. Age Ageing 35, ii12-ii18.

Masuda, K., Kim, J., Kinugasa, R., Tanabe, K., Kuno, SY., 2002. Determinants for stair climbing by elderly from muscle morphology. Percept. Mot. Skills 94, 814-816. 
Miyatani, M., Kanehisa, H., Ito, M., Kawakami, Y., Fukunaga, T., 2004. The accuracy of volume estimates using ultrasound muscle thickness measurements in different muscle groups. Eur. J. Appl. Physiol. 91, 264-272.

Morse, C.I., Thom, J.M., Birch, K.M., Narici, M.V., 2005. Changes in triceps surae muscle architecture with sarcopenia. Acta. Physiol. Scand. 183, 291-298.

Oguri, K., Zhao, L., Du, N., Kato, Y., Miyamoto, K., Hayakawa, M., Kurokawa, J., Nagasaki, S., Matsuoka, T., 2004. Association of habitual long-distance running with the thickness of skeletal muscles and subcutaneous fat in the body extremities and trunk in middle-aged men. J. Sports Med. Phys. Fitness 44, 417-423.

Park, H., Park, S., Shephard, R.J., Aoyagi, Y., 2010. Yearlong physical activity and sarcopenia in older adults: the Nakanojo Study. Eur. J. Appl. Physiol. 25, [Epub ahead of print] 
Reeves, N.D., Maganaris, C.N., Narici, M.V., 2004. Ultrasonographic assessment of human skeletal muscle size. Eur. J. Appl. Physiol. 91, 116-118.

Reimers, C.D., Harder, T., Saxe, H., 1998. Age-related muscle atrophy does not affect all muscles and can partly be compensated by physical activity: an ultrasound study. J. Neurol. Sci. 159, 60-66.

Roos, M.R., Rice, C.L., Vandervoort, A.A., 1997. Age-related changes in motor unit function. Muscle Nerve 20, 679-690.

Sanada, K., Kearns, C., Midorikawa, T., Abe, T., 2006. Prediction and validation of total and regional skeletal muscle mass by ultrasound in Japanese adults. Eur. J. Appl. Physiol. 96, 24-31.

Sandler, R.B., Burdett, R., Zaleskiewicz, M., Sprowls-Repcheck, C., Harwell, M., 1991. Muscle strength as an indicator of physical activity. Med. Sci. Sports Exerc. 23, $1375-1381$. 
Takahashi, K., Takahashi, H.E., Nakadaira, H., Yamamoto, M., 2006. Different changes of quantity due to aging in the psoas major and quadriceps femoris muscles in women. J. Musculoskelet. Neuronal Interact. 6, 201-205.

Thoirs, K., English, C., 2009. Ultrasound measures of muscle thickness: intra-examiner reliability and influence of body position. Clin. Physiol. Funct. Imaging 29, 440-446.

Tirosh, O., Sparrow, W.A., 2005. Age and walking speed effects on muscle recruitment in gait termination. Gait Posture 21, 279-288.

Walton, J.M., Roberts, N., Whitehouse, G.H., 1997. Measurement of the quadriceps femoris muscle using magnetic resonance and ultrasound imaging. Br. J. Sports Med. 31, 59-64.

Yazawa, A., Watanabe. K., Yoshida, Y., Imaki, M., Tanada, S., 2007. Relationship between physical activity measured by accelerometer and muscle mass, physical 
fitness and blood test values in middle-aged and elderly people. J. Analytical

Bio-Science 30, 279-285.

Young, A., Stokes, M., Crowe, M., 1985. The size and strength of the quadriceps muscles of old and young men. Clin. Physiol. 5, 145-154. 
Table 1.

Characteristics of the subjects, $n$, mean \pm S.D.,

Young group

Elderly group

Number

20

17

Age (years)

$19.8 \pm 0.8$

$83.2 \pm 6.2$

Height (cm)

$158.3 \pm 6.2$

$147.4 \pm 7.1$

Weight $(\mathrm{kg})$

$53.8 \pm 5.0$

$45.7 \pm 7.3$

$\mathrm{BMI}$

$21.5 \pm 1.7$

$21.1 \pm 3.7$ 
Table 2.

Measurement positions and measurement sites for each muscle

\begin{tabular}{|c|c|c|}
\hline Muscles & Positions & Measurement sites \\
\hline gluteus maximus & prone & $\begin{array}{l}30 \% \text { proximal between posterior superior iliac } \\
\text { spine and the greater trochanter }\end{array}$ \\
\hline gluteus medius & prone & $\begin{array}{l}\text { midway between the proximal end of iliac crest } \\
\text { and the greater trochanter }\end{array}$ \\
\hline gluteus minimus & prone & $\begin{array}{l}\text { midway between the proximal end of iliac crest } \\
\text { and the greater trochanter }\end{array}$ \\
\hline psoas major & prone & 7cm lateral from the $\mathrm{L} 3$ spinous process \\
\hline rectus femoris & supine & $\begin{array}{l}\text { midway between the anterior superior iliac } \\
\text { spine and the proximal end of the patella }\end{array}$ \\
\hline vastus lateralis & supine & $\begin{array}{l}3 \mathrm{~cm} \text { lateral of } 60 \% \text { distal between the anterior } \\
\text { superior iliac spine and the proximal end } \\
\text { of the patella }\end{array}$ \\
\hline vastus intermedius & supine & $\begin{array}{l}\text { midway between the anterior superior iliac spine } \\
\text { and the proximal end of the patella }\end{array}$ \\
\hline biceps femoris & prone & $\begin{array}{l}\text { midway between the ischial tuberosity and } \\
\text { the lateral condyle of the tibia }\end{array}$ \\
\hline gastrocnemius & prone & $\begin{array}{l}\text { medial head of gastrocnemius at } 30 \% \text { proximal } \\
\text { between the lateral malleolus of the fibula and the } \\
\text { lateral condyle of the tibia }\end{array}$ \\
\hline soleus & prone & $\begin{array}{l}30 \% \text { proximal between the lateral malleolus of } \\
\text { the fibula and the lateral condyle of the tibia }\end{array}$ \\
\hline
\end{tabular}


Table 3.

Comparison of muscle thickness $(\mathrm{mm})$ between the young and elderly groups, mean \pm S.D.

gluteus maximus

gluteus medius

gluteus minimus

psoas major

rectus femoris

vastus lateralis

vastus intermedius

biceps femoris

gastrocnemius

soleus

Notes: ${ }^{*}$ indicate significant differences at $p<0.01$
Young group

$25.0 \pm 2.98$

Elderly group

$15.5 \pm 3.85^{* *}$

$14.6 \pm 3.98^{* *}$

$12.1 \pm 4.11^{* *}$

$13.6 \pm 5.66^{\star *}$

$16.7 \pm 3.71^{\text {** }}$

$13.9 \pm 4.03^{* *}$

$21.5 \pm 3.52$

$16.1 \pm 3.79^{\text {** }}$

$36.5 \pm 4.87$

$17.9 \pm 4.81^{* *}$

$16.3 \pm 2.31$

$11.1 \pm 2.70^{* *}$

$34.5 \pm 6.11$

$29.7 \pm 7.90$ 
Table 4.

The rate $(\%)$ of muscle thickness in the elderly, meantS.D.

Muscles

gluteus maximus

$62.0 \pm 15.4$

gluteus medius

$63.7 \pm 17.4$

gluteus minimus

$62.8 \pm 21.3$

psoas major

$47.2 \pm 19.7$

rectus femoris

$72.9 \pm 16.2^{* *} \dagger$

vastus lateralis

$63.0 \pm 18.3$

vastus intermedius

$74.9 \pm 17.6^{\star *}+\dagger$

biceps femoris

$48.9 \pm 13.2$

gastrocnemius

$68.1 \pm 16.5$

soleus

$86.1 \pm 22.9^{* *}+\dagger$

Notes: ${ }^{* *} p<0.01$ significant difference compared to psoas major; ${ }^{\dagger \dagger} p<0.01,{ }^{\dagger} p<0.05$ significant difference compared to biceps femoris 
Table 5.

Relationship between muscle thickness and LSA in elderly women

\begin{tabular}{lll}
\hline & Correlation coeff. $(95 \% \mathrm{Cl})$ & $\mathrm{p}$ \\
\hline gluteus maximus & $0.197(-0.314-0.619)$ & 0.450 \\
gluteus medius & $0.528(0.064-0.805)$ & $0.029^{*}$ \\
gluteus minimus & $0.444(-0.047-0.762)$ & 0.075 \\
psoas major & $-0.194(-0.617-0.316)$ & 0.456 \\
rectus femoris & $-0.283(-0.672-0.229)$ & 0.271 \\
vastus lateralis & $-0.167(-0.599-0.341)$ & 0.522 \\
vastus intermedius & $-0.009(-0.488-0.474)$ & 0.973 \\
biceps femoris & $0.086(-0.411-0.544)$ & 0.742 \\
gastrocnemius & $-0.243(-0.648-0.269)$ & 0.347 \\
soleus & $0.329(-0.180-0.699)$ & 0.198
\end{tabular}

Notes: *indicates significant correlation at $p<0.05$ 